\title{
Beneficial effects of reading aloud and solving simple arithmetic calculations (learning therapy) on a wide range of cognitive functions in the healthy elderly: study protocol for a randomized controlled trial
}

Rui Nouchi ${ }^{1,2^{*}}$, Yasuyuki Taki ${ }^{3}$, Hikaru Takeuchi ${ }^{1}$, Hiroshi Hashizume ${ }^{3}$, Takayuki Nozawa ${ }^{1}$, Atsushi Sekiguchi ${ }^{4}$, Haruka Nouchi ${ }^{1}$ and Ryuta Kawashima ${ }^{1,3,4}$

\begin{abstract}
Background: Almost all cognitive functions decline with age. Results of previous studies have shown that cognitive training related to everyday life (reading aloud and solving simple arithmetic calculations), namely learning therapy, can improve two cognitive function (executive functions and processing speed) in elderly people. However, it remains unclear whether learning therapy engenders improvement of various cognitive functions or not. We investigate the impact of learning therapy on various cognitive functions (executive functions, episodic memory, short-term memory, working memory, attention, reading ability, and processing speed) in healthy older adults.

Methods: We use a single-blinded intervention with two parallel groups (a learning therapy group and a waiting list control group). Testers are blind to the study hypothesis and the group membership of participants. Through an advertisement in local newspaper, 64 healthy older adults are recruited. They will be assigned randomly to a learning therapy group or a waiting list control group. In the learning therapy group, participants are required to perform two cognitive tasks for 6 months: reading Japanese aloud and solving simple calculations. The waiting list group does not participate in the intervention. The primary outcome measure is the Stroop test score: a measure of executive function. Secondary outcome measures are assessments including the following: verbal fluency task, logical memory, first and second names, digit span forward, digit span backward, Japanese reading test, digit cancellation task, digit symbol coding, and symbol search. We assess these outcome measures before and after the intervention.
\end{abstract}

Discussion: This report is the first study which investigates the beneficial effects of learning therapy on a wide range of cognitive functions of elderly people. Our study provides sufficient evidence of learning therapy effectiveness. Most cognitive functions, which are correlated strongly with daily life activities, decrease with age. These study results can elucidate effects of cognitive training on elderly people.

Trial registration: This trial was registered in The University Hospital Medical Information Network Clinical Trials Registry (No. UMIN000006998).

\footnotetext{
* Correspondence: rnouchi@idac.tohoku.ac.jp

${ }^{1}$ Smart Ageing International Research Centre, Institute of Development,

Aging and Cancer, Tohoku University, Sendai 980-8575, Japan

Full list of author information is available at the end of the article
} 


\section{Background}

Cognitive function changes during a person's lifetime [1]. Elderly people might experience a decline in several cognitive functions such as memory [2], attention [3], executive functions $[4,5]$, and processing speed [6]. Decline in cognitive ability engenders difficulty in performing basic daily living activities [7-9]. Consequently, maintaining or improving cognitive function in older adults is drawing increasing attention [10-25].

Although human cognitive function typically declines with age, earlier studies showed that several cognitive training programs can improve cognitive functions such as memory $[18,26]$, processing speed $[21,27,28]$, executive function $[29,30]$, and attention [31] in healthy elderly people. It is particularly interesting that some studies have demonstrated that the effects of cognitive training can impact non-trained cognitive functions or tasks [30,32-35]. For instance, Schmiedek [35] conducted a working memory training study for elderly people in which participants were required to perform auditory recognition, discrimination, and memory tasks for about 15 min per day at least 5 days per week, for 8 to 10 weeks. Elderly people in the training group showed improvement of cognitive function in directly trained tasks (for example, alpha span and word list) and in cognitive functions in non-trained tasks (for example, animal span, rotation span, word pairs). Results of earlier studies show that cognitive training (for example working memory training) can improve cognitive functions in elderly people.

In line with previous studies using cognitive training for elderly people, we recently developed a new mode of cognitive training using reading aloud and solving of simple arithmetic calculations, namely learning therapy $[30,36]$. Learning therapy is designed for stimulation of the frontal cortex (especially dorsolateral prefrontal cortex) and of the temporal and parietal association cortices by cognitive tasks, thereby engendering improvement of the function of these cortices [36]. We specifically targeted these regions for the following reasons: (1) Previous functional magnetic resonance imaging (MRI) studies showed that task-related activation of these regions in older adults is lower than that in younger adults [37-42]; (2) Previous structural MRI studies using voxel-based morphometry (VBM) showed that regional gray and white matter volumes of these regions decline with age [43-45]; and (3) These activity and regional gray matter volumes of these regions are closely linked to cognitive functions $[39,46-52]$ such as executive functions, processing speed, and memory, which decrease with age. Therefore, cognitive decline in elderly people might result from reductions of activities and volume in these regions. Based on these facts, we assumed that stimulation of the frontal cortex (especially the dorsolateral prefrontal cortex), as well as those of the temporal and parietal association cortices by cognitive tasks might improve activities and regional gray matter volumes of these cortices. Moreover, they might engender improvement of the functions of these cortices $[10,11,18,36,53]$.

Learning therapy used two simple and easy training tasks (reading Japanese aloud and solving simple arithmetic calculations) derived from knowledge of neuroscience. Results of brain imaging studies indicate that reading sentences or words aloud [54-58] and simple arithmetic operations [59-61] activate the three associated cortices, especially the prefrontal cortex. Reading aloud is accomplished using a combination of several cognitive processes such as recognition of visually presented words, conversion to phonological representation from graphic representation of words, analysis of the meaning of words, and control of pronunciation. Solving arithmetic problems is also accomplished through the use of numerous cognitive processes such as recognition of visually presented numbers, arithmetic operations, and control of hand movements. Moreover, the bilateral prefrontal cortices are activated even when solving very simple and easy problems. Both reading aloud and solving arithmetic problems require working memory. This prefrontal stimulation might engender the positive transfer effect on other cognitive functions. Learning therapy has outstanding features compared to previous cognitive training. First, training tasks of learning therapy are based on results of neuroscience. Secondly, the training tasks are extremely simple and easy for elderly people to perform. Consequently, elderly people can readily comprehend and perform training tasks.

Previous studies using learning therapy have demonstrated that learning therapy can improve executive functions and processing speed in healthy elderly people. For instance, Uchida and Kawashima [30] conducted a randomized controlled trial using learning therapy for healthy elderly people. Participants were divided into learning therapy and control groups. The learning therapy group was required to do two training tasks for 5 days a week: reading Japanese aloud and conducting simple calculations. After 6 months, the learning therapy group showed improved scores in the frontal assessment battery (FAB at bedside), which measures executive function [62-64], and a digit-symbol substitution test, which measures processing speed [65]. These results suggest that learning therapy beneficially affects some cognitive functions in elderly people.

\section{Purpose of this study}

An earlier study showed effects of learning therapy transferred to executive functions and processing speed [30]. However, it remains unclear whether or not the 
effects of learning therapy can transfer (improve) other cognitive functions such as memory and attention in elderly people. Consequently, the purpose of this study is to investigate whether or not learning therapy can transfer to a wide range of cognitive functions in elderly people. To reveal transfer effects of learning therapy on cognitive functions, we conduct a single-blinded randomized control trial using learning therapy. Testers are blinded to the study hypothesis and the group membership of participants. To evaluate the transfer effects of the reading aloud and solving simple arithmetic calculations interventions (learning therapy), we assess a broad range of cognitive functions. The measured cognitive functions are divisible into seven categories: executive functions, episodic memory, short-term memory, working memory, reading ability, attention, and processing speed.

\section{Method}

\section{Randomized controlled trial design and setting of this} trial

This study, which was registered in the University Hospital Medical Information Network (UMIN) Clinical Trial Registry (UMIN000006998), is a randomized controlled trial conducted in Sendai city, Miyagi prefecture, Japan. Written informed consent to participate in the study will be obtained from each participant before enrolment. The protocol of this study and informed consent were approved by the Ethics Committee of the Tohoku University Graduate School of Medicine.

To assess the impact of learning therapy on a wide range of cognitive functions in healthy elderly people, we use a single-blinded intervention with two parallel groups: a learning therapy group and a waiting list control group. Testers are blind to the study's hypothesis and the group membership of participants. The Consolidated Standards of Reporting Trials (CONSORT) statement [66]http://www.consort-statement.org/home/ has been used as a framework for developing the study methodology (Additional file 1). The trial design is shown in Figure 1.

\section{Recruitment and selection of participants}

Participants are recruited from the general population through advertisements in the local town paper and local newspaper. Interested participants are screened using a semi-structured telephone interview. After the telephone interview, participants are invited to visit Tohoku University for a more detailed screening assessment and to provide written informed consent.

\section{Inclusion and exclusion criteria}

The purpose of this intervention is to investigate transfer effects of learning therapy for a range of cognitive functions in healthy older adults. The criteria include participants who report themselves to be right-handed, native Japanese speakers, unconcerned about their own memory functions, not using medications known to interfere with cognitive functions (including benzodiazepines, antidepressants or other central nervous agents), and having no disease known to affect the central nervous system, including thyroid disease, multiple sclerosis, Parkinson disease, stroke, severe hypertension (systolic blood pressure is over 180, diastolic blood pressure is over 110), and diabetes. Age of participants is over 65 years old. Criteria exclude participants who have an Intelligence Quotient (IQ) less than 85 derived from Japanese Reading Test (JART) [67]. Participants who will participate in another cognitive-related intervention studies will be excluded.

\section{Randomization}

Randomization is designed to take place after receiving the informed consent statement. A researcher (RN) with no contact with the study participants randomly assigns participants to either the learning therapy or the waiting list group by random draw using an online computer program http://www.graphpad.com/quickcalcs/index. $\mathrm{cfm}$. Letters are used to inform participants of their allocation.

\section{Learning therapy group (cognitive intervention group)}

The cognitive intervention method is the same as that used in our previous study using learning therapy for healthy older adults [30]. Training tasks use two simple tasks (solving arithmetic and Japanese language problems) that are systematized basic problems in arithmetic and reading $[30,36]$. We prepared various materials used in everyday classes of first-grade to fourth-grade elementary school students. The problems are printed on both sides of an A4 sheet of paper $(210 \times 297 \mathrm{~mm})$. For the arithmetic problems, the lowest level of difficulty is single-digit addition. The highest level is three-digit division. For the Japanese language problems, the lowest level of difficulty is reading and writing simple sentences. The highest level is reading fairy tales aloud.

One or two weeks before the start of the intervention program, intervention group participants are asked to go to a classroom. The appropriate level of difficulty and workload for all participants is assessed by diagnostic tests, which consist of 70 arithmetic and 16 language problems. The arithmetic problems range in difficulty from single-digit addition to three-digit division. The language problems range in difficulty from reading and comprehending Japanese Haiku (17 characters) to reading and comprehending stories (110 characters). For tests of both types, the percentage of correct answers and the time it takes to solve all problems are 


\section{CONSORT diagram}

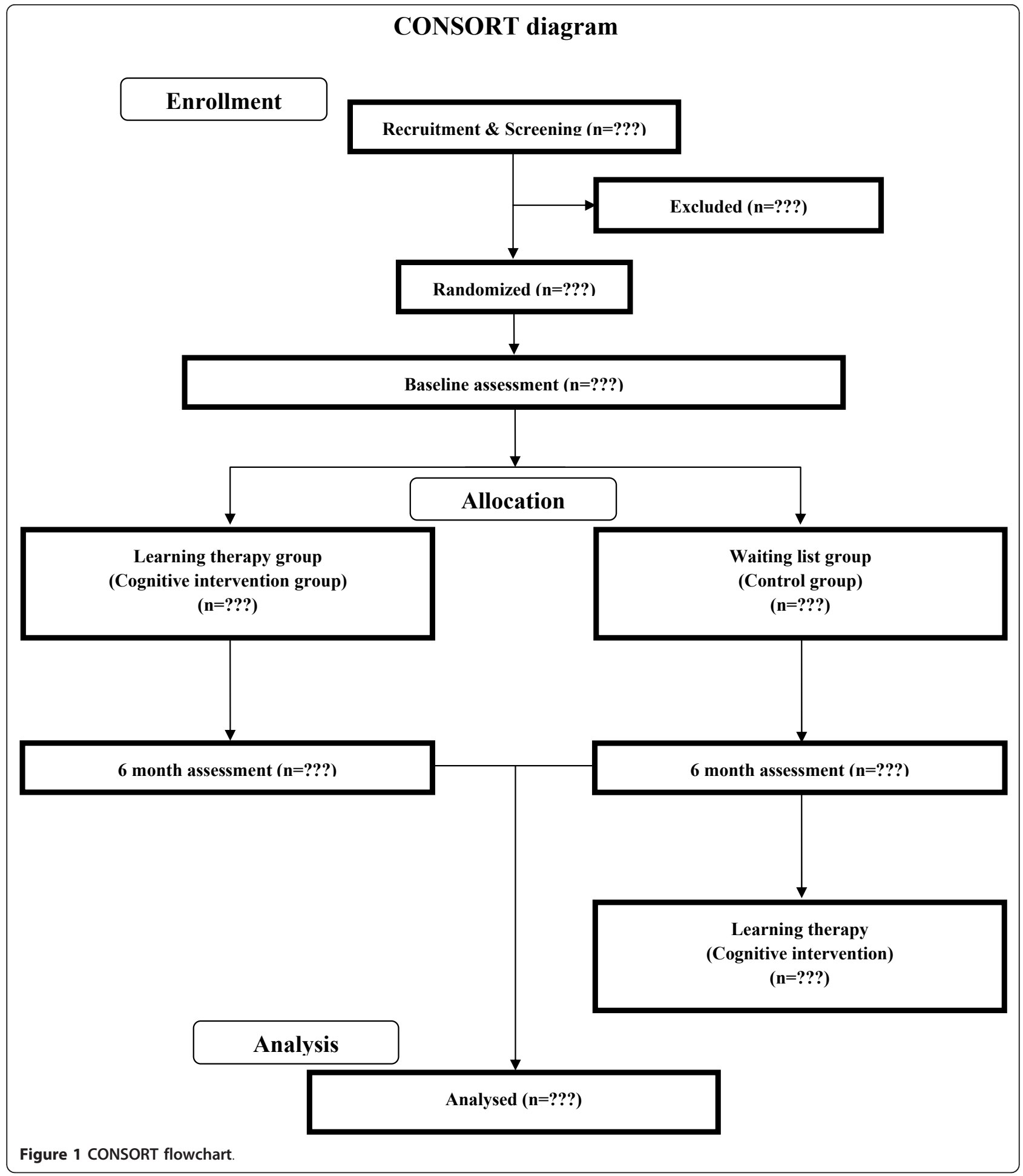

determined. In this intervention, the difficulty level and workload of each task are set such that each participant is able to solve the problems with ease and without mental stress within 15 min.

The cognitive intervention is scheduled to be conducted for 23 weeks. Participants in the cognitive intervention group are asked to go to the classroom in Tohoku University once a week. They are instructed to complete five sheets of each task prepared for each for that day, which are assessed by staff members. Mistakes are corrected by the participants themselves. The study period ends when the participants complete each of the 
problems correctly. The daily learning time for the two tasks is approximately $15 \mathrm{~min}$. The cognitive intervention is undertaken on an individual basis, so that the participants can decide how to use their learning time of 15 min freely. Participants are also asked to do their homework of two tasks for 4 to 6 days a week. Regarding their homework, participants are asked to complete five sheets of each task prepared for each. Furthermore, participants are asked to bring back their achievements of homework on the next school day. The staff members check their homework and provide advice when necessary.

\section{Waiting list group (no cognitive intervention group)}

The wait-listed group receives no intervention. Those participants are informed by letter that they are scheduled to receive an invitation to participate after a waiting period of 6 months. No placebo is used for the social contact group. Results of previous intervention studies $[26,68]$ report that a placebo group is unnecessary for this type of study because no difference exists in cognitive or functional improvement between the placebo and no-social-contact groups (control group).

\section{Overview of cognitive function measures}

To evaluate the beneficial effects of learning therapy on cognitive functions, we assess a broad range of cognitive functions (Table 1). Measures of the cognitive functions are divisible into seven categories (executive functions, episodic memory, short-term memory, working memory, reading ability, attention, and processing speed). Executive functions are measured using the Stroop test (ST) [69] and verbal fluency task (VFT) [70]. Episodic memory is measured using logical memory (LM) [71] and first and second names (FS-N) [72]. Short-term memory is measured using digit span forward (DS-F) [65]. Working memory is measured using digit span backward (DSB) [65]. Reading ability is measured using the Japanese reading test (JART) [67]. Attention is measured using

Table 1 Summary of cognitive function measures

\begin{tabular}{ll}
\hline Cognitive function & Task \\
\hline Executive functions & Stroop test \\
\hline Episodic memory & Verbal fluency task \\
\hline & Logical memory \\
\hline Short-term memory & First and second names \\
\hline Working memory & Digit span forward \\
\hline Reading ability & Digit span backward \\
\hline Attention & Japanese reading test \\
\hline Processing speed & Digit cancellation task \\
\hline & Digit symbol coding \\
\hline
\end{tabular}

the digit cancellation task (D-CAT) [73]. Processing speed is measured using digit symbol coding (Cd) [65] and symbol search (SS) [65]. Details of all tasks are described below.

We assess these cognitive function measures before and after the intervention period (6 months). The primary outcome measure is ST. We selected ST as the primary outcome measure because: (1) learning therapy is expected to improve executive functions, and a previous study showed learning therapy can improve executive function measured by FAB [30]; (2) ST is a task that is often used to measure executive functions [74,75]; and (3) ST has been standardized, with high reliability and validity in Japanese populations $[69,76]$.

ST

Stroop test (ST) measures executive function including response inhibition and impulsivity. Hakoda's version is a paper and pencil version ST [69]. In this test, participants must check whether their chosen answers are correct, unlike the traditional oral naming ST. We use a reverse ST and a ST. In the reverse ST, in the leftmost of six columns, a word naming a color is printed in another color (for example 'red' is printed in blue letters); the other five columns are each filled with five different colors from which participants must check the column whose color matches the written word in the leftmost column. In the $\mathrm{ST}$, in the leftmost of six columns, a word naming a color is printed in another color (for example 'red' is printed in blue letters) and the other five columns contain words naming colours. Participants must check the column containing the word naming the color of the word in the leftmost column. In each task, participants are instructed to complete as many of these exercises as possible in $1 \mathrm{~min}$. The primary measure for this task is the number of correct items.

VFT

Verbal fluency task (VFT) measures executive function. We use the Japanese version of VFT [70], which has two tasks (letter fluency task (LFT) and category fluency (CFT) task). In LFT, a Japanese letter, 'ka', is given to each participant, who is then asked to generate common nouns beginning with this letter - as many as possible in $60 \mathrm{~s}$. In CFT, a category name (animal) is given to each participant, who is then asked to generate many words of a certain category (animal). The participants are instructed not to include proper nouns or to repeat one that has already been stated. The primary measure for this task is the number of words reported. The reliability and validity of Japanese LFT were demonstrated by Ito [70]. LM

Logical memory (LM) evaluates the performance of episodic memory. LM is a subtest of the Wechsler Memory Scale-Revised (WMS-R) [71]. LM consists of two short paragraph-length stories (Story A and Story B). In LM, 
participants must memorize the short story. The stories are scored in terms of the number of story units recalled, as specified in the WMS-R scoring protocol. We use either Story A or Story B. The primary measure for this task is the number of correct story units recalled.

\section{FSN}

First and second names (FSN) evaluates memory ability in everyday life. FSN is a subset of Rivermead Behavioral Memory Test (RBMT) [72]. RBMT measures episodic memory as it is used in everyday life. Therefore, subsets of RBMT are similar to everyday situations. In FSN, participants must memorize first and second names with faces (photograph). Subsequently, they must recall the first and the second names when the face is shown again later. We use four faces (four first names and four second names). The primary measure of this test is the total number of correct answers in both first and second names. The maximum raw score of FSN is 8 .

\section{DS}

Digit span (DS) is a subtest in Wechsler Adult Intelligence Scale-Third Edition (WAIS-III) [65]. DS, which has two subsections (DS-F and DS-B), evaluates shortterm memory and working memory. DS-F measures short-term memory by simply requiring participants to repeat numbers. DS-B measures working memory by requiring participants to memorize numbers and repeat the numbers in the inverse order. For DS-F, participants repeat numbers in the same order as they were read aloud by the examiner. For DS-B, participants repeat numbers in the reverse order of that presented aloud by the examiner. In both, the examiner reads a series of number sequences which the examinee must repeat in either forward or reverse order. DS-F has 16 sequences. DS-B has 14 sequences. The primary measures of this test are raw scores that reflect the number of correctly repeated sequences until the discontinue criterion (that is, failure to reproduce two sequences of equal length) is met [65]. The maximum raw score of DS-F is 16. The maximum raw score of DS-B is 14 .

\section{JART}

The Japanese reading test (JART) measures reading ability [67]. JART is a Japanese version of the National Adult Reading Test (NART) which has a reading test of 50 irregularly spelled words in English (for example ache) [77]. JART is a reading test comprising $25 \mathrm{Kanji}$ compound words (e.g. $\nabla \nabla, \nabla \nabla)$. The reading stimuli are printed out randomly for reading. The participants are asked to read each Kanji compound word aloud. This task assesses reading ability and IQ. The primary measure for this task is the number of correct items.

\section{$D-C A T$}

Digit cancellation task (D-CAT) evaluates attention [73]. The test sheet consists of 12 rows of 50 digits. Each row contains five sets of numbers 0 to 9 arranged in random order. Consequently, any one digit appears five times in each row with randomly determined neighbours. DCAT consists of three such sheets. Participants are instructed to search for the target number(s) that had been specified to them and to delete each one with a slash mark as quickly and as accurately as possible until the experimenter sends a stop signal. There are three trials, first with a single target number (6), second with two target numbers (9 and 4$)$, and third with three $(8,3$, and 7). Each trial is given for $1 \mathrm{~min}$. Consequently, the total time required for $\mathrm{D}-\mathrm{CAT}$ is $3 \mathrm{~min}$. In the second and third trials, it is emphasized that all the target numbers instructed should be cancelled without omission. The primary measure of this test is the number of hits (correct answers). We use only the number of hits in the first trial.

\section{Cd}

Digit symbol coding (Cd) is a subtest of WAIS-III [65]. This test measures processing speed. For $\mathrm{Cd}$, the participants are shown a series of symbols that are paired with numbers. Using a key within a $120 \mathrm{~s}$ time limit, participants draw each symbol under its corresponding number. The primary measure of this test is the number of correct answers.

SS

Symbol search (SS), a subtest of WAIS-III containing 60 items [65], measures processing speed. For this subtest, participants visually scan two groups of symbols (a target group and a search group) and report whether either of the target symbols matches any symbol in the search group. Participants respond to as many items as possible within a $120 \mathrm{~s}$ time limit. The primary measure of this test is the number of correct answers.

\section{Sample size}

Our sample size estimation is based on the change score in the reverse $\mathrm{ST}$, which is the primary outcome in this study. We expected to detect a large effect size $\left(\eta^{2}=\right.$ 0.14) of the change score in the reverse ST between learning therapy and waiting list groups. the sample size was determined using $G$ * power [78,79] based on $80 \%$ power, a two-sided hypothesis test, an alpha level of $5 \%$, an analysis of covariance (ANCOVA) model that includes a baseline reverse Stroop task score, age, and sex as a covariate. The sample size calculation indicated that we need 32 participants in each of the learning therapy and waiting list groups with consideration of a $20 \%$ drop-out rate.

\section{Analysis}

This study is designed to evaluate the beneficial effect of learning therapy in elderly people. We calculate the change score (post-training score minus pre-training 
score) in all cognitive function measures. We conduct an ANCOVA for the change scores in each cognitive test. The change scores are the dependent variable. Groups (learning therapy, waiting list) are the independent variable. Pre-training scores in the dependent variable, sex, age categories are the covariates to exclude the possibility that any pre-existing difference of measure between groups affect the result of each measure and to adjust for background characteristics. The level of significance is set at $\mathrm{p}<0.05$. Moreover, we report eta squared $\left(\eta^{2}\right)$ as an index of effect size. It is the standardized difference in the change score between intervention groups (learning therapy group, waiting list group). In actuality, $\eta^{2} \geq 0.01$ is regarded as a small effect, $\eta^{2} \geq .006$ as a medium effect, and $\eta^{2} \geq 0.14$ as a large effect [80]. Missing data are imputed using the expectation-maximization method, as implemented in the Statistical Package for the Social Sciences (SPSS) Missing Value Analysis. It imputes missing values using maximum likelihood estimation with the observed data in an iterative process [81]. All randomized participants are included in the analyses in line with their allocation, irrespective of how many sessions they complete (intention-to-treat principle). All analyses are performed using SPSS software (ver. 18 or higher).

\section{Discussion}

This study is designed to investigate the beneficial effects of learning therapy on widely various cognitive functions such as executive functions, episodic memory, short-term memory, working memory, reading ability, attention, and processing speed in healthy elderly people.

This study has several strengths compared to earlier studies using cognitive training for elderly people. First, this study was designed according to CONSORT guidelines [66] http://www.consort-statement.org/consortstatement/, which are intended to improve standards of reporting of randomized clinical trials (RCT). Consequently, this study has been structured to enable its reproduction in both research and clinical settings. Moreover, we can provide sufficient evidence of the effectiveness of cognitive training such as learning therapy.

Second, we investigate the beneficial effects of learning therapy on widely various cognitive functions. The measures assess cognitive functions of seven categories: executive functions, episodic memory, short-term memory, working memory, reading ability, attention, and processing speed. Various cognitive functions are necessary to support our actions and behaviours in everyday life. For instance, when we cook meals, we must: (1) choose a menu (executive functions); (2) remember the refrigerator contents (memory); (3) seek and select seasonings from storage (attention); and (4) cut and prepare cooking ingredients with speed and efficiency (processing speed). Because of the complexity of such an apparently simple task, it is expected to be important to investigate the beneficial effects of learning therapy on widely various cognitive functions.

Third, we use simple, easily learned training tasks (reading aloud and simple calculation) using paper and pencil. Most training tasks in previous studies were complex tasks using computers [18,31,82-84]. Using computers might make it easy to record data precisely and to control tasks. Nevertheless, elderly people often have difficulty using computers [85-87]. The difficulty using computers might cause frustration and other negative emotion, possibly reducing their motivation to continue. Our training tasks are more familiar to elderly people and thus expected to encourage their willingness.

This study has some limitations. A first limitation is the intervention period. Our intervention period is about 6 months. Some previous studies have shown that short-term intervention (four example 4 to 6 weeks) improved cognitive function in elderly people $[18,20]$. Considering reduced costs for elderly people, shorter intervention studies using learning therapy would be also needed. A second limitation is participants. We recruit only healthy elderly people for participation in this study. Providing the validity of effects of learning therapy on widely various cognitive functions, we must conduct the same randomized controlled trial (RCT) for non-healthy elderly people such as those with dementia or depression.

In summary, this study is the first to reveal the beneficial effects of learning therapy on a wide range of cognitive functions in elderly people. Our study is designed to provide sufficient evidence of effectiveness of learning therapy. Given that most cognitive functions decrease with age [1] and that these functions are strongly correlated with daily life activities [7-9], our results can elucidate the effects of cognitive training for elderly people.

\section{Trial status}

Recruitment of participants begins in February 2012, and is expected to end in January 2013.

\section{Additional material}

Additional file 1: CONSORT 2010 checklist of information to include when reporting a randomized trial*

\section{Abbreviations}

ANCOVA: Analysis of covariance; Cd: Digit symbol coding; CONSORT: Consolidated standards of reporting trials; D-CAT: Digit cancellation task; DSB: Digit span backward; DS-F: Digit span forward; FS-N: First and second 
names; IQ: Intelligence quotient; JART: Japanese reading test; LM: Logical memory; MRI: Magnetic resonance imaging; RCT: Randomized controlled trial; RBMT: Rivermead behavioral memory test; SPSS: Statistical package for the social sciences; SS: Symbol search; ST: Stroop test; UMIN: University Hospital Medical Information Network; VBM: Voxel based morphometry; VFT: Verbal fluency task; WAIS-III: Wechsler Adult Intelligence Scale-Third Edition; WMS-R: Wechsler memory scale-revised.

\section{Acknowledgements}

Ethical approval was provided by the Institutional Review Board of the Tohoku University Graduate School of Medicine (ref. 2011-153). Based on the Declaration of Helsinki, written informed consent will be received from each participant. This study is one industry-academy collaboration of Tohoku University, namely Smart Aging Square http://www2.idac.tohoku.ac.jp/dep/ sairc/square.html. This study is supported by the KUMON Institute of Education and a Grant-in-Aid from the Japan Society for the Promotion of Science (JSPS) for Fellows (grant no. 235019; http://www.jsps.go.jp/english/e$\mathrm{pd} /$ index.html). Funding sources of the trial have no involvement in the study design, collection, analysis, interpretation of data, or writing of papers. We thank A. Kasagi for recruiting the participants, testers for performing psychological tests, supporters for conducting learning therapy, the participants, and all our other colleagues in IDAC, Tohoku University for their support.

\section{Author details}

${ }^{1}$ Smart Ageing International Research Centre, Institute of Development, Aging and Cancer, Tohoku University, Sendai 980-8575, Japan. ${ }^{2}$ Japanese Society for the Promotion of Science, Tokyo 102-8472, Japan. ${ }^{3}$ Division of Developmental Cognitive Neuroscience, Institute of Development, Aging and Cancer, Tohoku University, Sendai 980-8575, Japan. ${ }^{4}$ Department of Functional Brain Imaging, Institute of Development, Aging and Cancer, Tohoku University, Sendai 980-8575, Japan.

\section{Authors' contributions}

RN designed, developed the study protocol, and calculated the sample size. RN and HN searched the literature, selected cognitive function measures, created manuals to conduct and rate cognitive measures, and recruited testers for cognitive function measures. HN conducts cognitive function measures and rates these cognitive function measures with testers. RN supervises testers. RN wrote the manuscript with $Y T, H T, H H, Y N, A S, H N$, and RK. RK also gave advice related to the study protocol. All authors read and approved the final manuscript.

\section{Competing interests}

Learning therapy was developed by RK and KUMON Institute of Education. However, RK derives no income from KUMON Institute of Education and Society for Learning Therapy. RK has no other competing interests. All other authors have declared no competing interests.

\section{Received: 10 February 2012 Accepted: 6 April 2012}

Published: 6 April 2012

\section{References}

1. Hedden T, Gabrieli JD: Insights into the ageing mind: a view from cognitive neuroscience. Nat Rev Neurosci 2004, 5:87-96.

2. Salthouse TA: Memory aging from 18 to 80 . Alzheimer Dis Assoc Disord 2003, 17:162-167.

3. Yakhno NN, Zakharov W, Lokshina AB: Impairment of memory and attention in the elderly. Neurosci Behav Physiol 2007, 37:203-208.

4. Royall DR, Palmer R, Chiodo LK, Polk MJ: Declining executive control in normal aging predicts change in functional status: The Freedom House Study. J Am Geriatr Soc 2004, 52:346-352.

5. Coppin AK, Shumway-Cook A, Saczynski JS, Patel KV, Ble A, Ferrucci L, Guralnik JM: Association of executive function and performance of dualtask physical tests among older adults: analyses from the InChianti study. Age Ageing 2006, 35:619-624

6. Salthouse TA: The processing-speed theory of adult age differences in cognition. Psychol Rev 1996, 103:403-428.

7. Cahn-Weiner DA, Malloy PF, Boyle PA, Marran M, Salloway S: Prediction of functional status from neuropsychological tests in community-dwelling elderly individuals. Clin Neuropsychol 2000, 14:187-195.
8. Lee Y, Kim JH, Lee K, Han G, Kim JL: Association of cognitive status with functional limitation and disability in older adults. Aging Clin Exp Res 2005, 17:20-28.

9. Owsley C, McGwin G Jr: Association between visual attention and mobility in older adults. J Am Geriatr Soc 2004, 52:1901-1906.

10. Fernandez-Prado S, Conlon S, Mayan-Santos JM, Gandoy-Crego M: The influence of a cognitive stimulation program on the quality of life perception among the elderly. Arch Gerontol Geriatr 2012, 54:181-184.

11. Tardif S, Simard M: Cognitive stimulation programs in healthy elderly: a review. Int J Alzheimers Dis 2011, 2011:378934.

12. Martin M, Clare L, Altgassen AM, Cameron MH, Zehnder F: Cognition-based interventions for healthy older people and people with mild cognitive impairment. Cochrane Database Syst Rev 2011, CD006220.

13. Williams KN, Kemper S: Interventions to reduce cognitive decline in aging. J Psychosoc Nurs Ment Health Serv 2010, 48:42-51.

14. Mowszowski L, Batchelor J, Naismith SL: Early intervention for cognitive decline: can cognitive training be used as a selective prevention technique? Int Psychogeriatr 2010, 22:537-548.

15. Lovden M, Backman L, Lindenberger U, Schaefer S, Schmiedek F: A theoretical framework for the study of adult cognitive plasticity. Psychol Bull 2010, 136:659-676.

16. Zelinski EM: Far transfer in cognitive training of older adults. Restor Neurol Neurosci 2009, 27:455-471.

17. Tucker-Drob EM, Johnson KE, Jones RN: The cognitive reserve hypothesis: a longitudinal examination of age-associated declines in reasoning and processing speed. Dev Psychol 2009, 45:431-446.

18. Smith GE, Housen P, Yaffe K, Ruff R, Kennison RF, Mahncke HW, Zelinski EM: A cognitive training program based on principles of brain plasticity: results from the Improvement in Memory with Plasticity-based Adaptive Cognitive Training (IMPACT) study. J Am Geriatr Soc 2009, 57:594-603.

19. Bissig D, Lustig C: Who benefits from memory training? Psychol Sci 2007, 18:720-726.

20. Willis SL, Tennstedt SL, Marsiske M, Ball K, Elias J, Koepke KM, Morris JN, Rebok GW, Unverzagt FW, Stoddard AM, Wright E: Long-term effects of cognitive training on everyday functional outcomes in older adults. JAMA 2006, 296:2805-2814

21. Ball K, Berch DB, Helmers KF, Jobe JB, Leveck MD, Marsiske M, Morris JN, Rebok GW, Smith DM, Tennstedt SL, et al: Effects of cognitive training interventions with older adults: a randomized controlled trial. JAMA 2002, 288:2271-2281.

22. Simoes $A$ : The effects of a cognitive training on mobility of elderly people. Stud Health Technol Inform 1998, 48:369-373.

23. Baltes $P B$, Sowarka $D$, Kliegl $R$ : Cognitive training research on fluid intelligence in old age: what can older adults achieve by themselves? Psychol Aging 1989, 4:217-221.

24. Willis SL: Cognitive training and everyday competence. Annu Rev Gerontol Geriatr 1987, 7:159-188.

25. Schaie KW, Willis SL, Hertzog C, Schulenberg JE: Effects of cognitive training on primary mental ability structure. Psychol Aging 1987, 2:233-242.

26. Mahncke HW, Connor BB, Appelman J, Ahsanuddin ON, Hardy JL, Wood RA, Joyce NM, Boniske T, Atkins SM, Merzenich MM: Memory enhancement in healthy older adults using a brain plasticity-based training program: a randomized, controlled study. Proc Natl Acad Sci USA 2006, 103:12523-12528.

27. Ball K, Edwards JD, Ross LA: The impact of speed of processing training on cognitive and everyday functions. J Gerontol B Psychol Sci Soc Sci 2007, 62:19-31.

28. Edwards JD, Wadley VG, Vance DE, Wood K, Roenker DL, Ball KK: The impact of speed of processing training on cognitive and everyday performance. Aging Ment Health 2005, 9:262-271.

29. Nouchi R, Taki Y, Takeuchi H, Hashizume H, Akitsuki Y, Shigemune $Y$, Sekiguchi A, Kotozaki Y, Tsukiura T, Yomogida Y, Kawashima R: Brain training game improves executive functions and processing speed in the elderly: A randomized controlled trial. PLoS One 2012, 7:e29676.

30. Uchida S, Kawashima R: Reading and solving arithmetic problems improves cognitive functions of normal aged people: a randomized controlled study. Age (Dordr) 2008, 30:21-29.

31. Mozolic JL, Long AB, Morgan AR, Rawley-Payne M, Laurienti PJ: A cognitive training intervention improves modality-specific attention in a 
randomized controlled trial of healthy older adults. Neurobiol Aging 2011, 32:655-668.

32. Buschkuehl M, Jaeggi SM, Hutchison S, Perrig-Chiello P, Dapp C, Muller M, Breil F, Hoppeler H, Perrig WJ: Impact of working memory training on memory performance in old-old adults. Psychol Aging 2008, 23:743-753.

33. Dahlin E, Nyberg L, Backman L, Neely AS: Plasticity of executive functioning in young and older adults: immediate training gains, transfer, and long-term maintenance. Psychol Aging 2008, 23:720-730.

34. Richmond LL, Morrison AB, Chein JM, Olson IR: Working memory training and transfer in older adults. Psychol Aging 2011, 26:813-822.

35. Schmiedek F, Lovden M, Lindenberger U: Hundred days of cognitive training enhance broad cognitive abilities in adulthood: findings from the COGITO Study. Front Aging Neurosci 2010, 2:27.

36. Kawashima R, Okita K, Yamazaki R, Tajima N, Yoshida H, Taira M, Iwata K, Sasaki T, Maeyama K, Usui N, Sugimoto K: Reading aloud and arithmetic calculation improve frontal function of people with dementia. J Gerontol A Biol Sci Med Sci 2005, 60:380-384.

37. Cabeza R: Cognitive neuroscience of aging: contributions of functional neuroimaging. Scand J Psychol 2001, 42:277-286.

38. Cabeza R, Daselaar SM, Dolcos F, Prince SE, Budde M, Nyberg L: Taskindependent and task-specific age effects on brain activity during working memory, visual attention and episodic retrieval. Cereb Cortex 2004, 14:364-375.

39. Eyler LT, Sherzai A, Kaup AR, Jeste DV: A review of functional brain imaging correlates of successful cognitive aging. Biol Psychiatry 2011, 70:115-122.

40. Grady CL, Mclntosh AR, Craik FI: Task-related activity in prefrontal cortex and its relation to recognition memory performance in young and old adults. Neuropsychologia 2005, 43:1466-1481.

41. Grady CL, Springer MV, Hongwanishkul D, McIntosh AR, Winocur G: Agerelated changes in brain activity across the adult lifespan. $J$ Cogn Neurosci 2006, 18:227-241.

42. Spreng RN, Wojtowicz M, Grady CL: Reliable differences in brain activity between young and old adults: a quantitative meta-analysis across multiple cognitive domains. Neurosci Biobehav Rev 2010, 34:1178-1194.

43. Bartzokis G, Beckson M, Lu PH, Nuechterlein KH, Edwards N, Mintz J: Agerelated changes in frontal and temporal lobe volumes in men: a magnetic resonance imaging study. Arch Gen Psychiatry 2001, 58:461-465.

44. Draganski B, Ashburner J, Hutton C, Kherif F, Frackowiak RS, Helms G, Weiskopf N: Regional specificity of MRI contrast parameter changes in normal ageing revealed by voxel-based quantification (VBQ). Neuroimage 2011, 55:1423-1434.

45. Giorgio A, Santelli L, Tomassini V, Bosnell R, Smith S, De Stefano N, Johansen-Berg $\mathrm{H}$ : Age-related changes in grey and white matter structure throughout adulthood. Neuroimage 2010, 51:943-951.

46. Bendlin BB, Fitzgerald ME, Ries ML, Xu G, Kastman EK, Thiel BW, Rowley HA, Lazar M, Alexander AL, Johnson SC: White matter in aging and cognition: a cross-sectional study of microstructure in adults aged eighteen to eighty-three. Dev Neuropsychol 2010, 35:257-277.

47. Brickman AM, Habeck C, Zarahn E, Flynn J, Stern Y: Structural MRI covariance patterns associated with normal aging and neuropsychological functioning. Neurobiol Aging 2007, 28:284-295.

48. Brickman AM, Zimmerman ME, Paul RH, Grieve SM, Tate DF, Cohen RA, Williams LM, Clark CR, Gordon E: Regional white matter and neuropsychological functioning across the adult lifespan. Biol Psychiatry 2006, 60:444-453.

49. Chee MW, Chen KH, Zheng H, Chan KP, Isaac V, Sim SK, Chuah LY, Schuchinsky M, Fischl B, Ng TP: Cognitive function and brain structure correlations in healthy elderly East Asians. Neuroimage 2009, 46:257-269.

50. Grady CL: Functional brain imaging and age-related changes in cognition. Biol Psychol 2000, 54:259-281.

51. Kaup AR, Mirzakhanian H, Jeste DV, Eyler LT: A review of the brain structure correlates of successful cognitive aging. I Neuropsychiatry Clin Neurosci 2011, 23:6-15.

52. Zimmerman ME, Brickman AM, Paul RH, Grieve SM, Tate DF, Gunstad J, Cohen RA, Aloia MS, Williams LM, Clark CR, Whitford TJ, Gordon E: The relationship between frontal gray matter volume and cognition varies across the healthy adult lifespan. Am J Geriatr Psychiatry 2006, 14:823-833.

53. Zelinski EM, Spina LM, Yaffe K, Ruff R, Kennison RF, Mahncke HW, Smith GE: Improvement in memory with plasticity-based adaptive cognitive training: results of the 3-month follow-up. J Am Geriatr Soc 2011, 59:258-265.

54. Graves WW, Desai R, Humphries C, Seidenberg MS, Binder JR: Neural systems for reading aloud: a multiparametric approach. Cereb Cortex 2010, 20:1799-1815.

55. Ino T, Nakai R, Azuma T, Kimura T, Fukuyama H: Recognition and reading aloud of kana and kanji word: an fMRI study. Brain Res Bull 2009, 78:232-239.

56. Parker Jones O, Green DW, Grogan A, Pliatsikas C, Filippopolitis K, Ali N, Lee HL, Ramsden S, Gazarian K, Prejawa S, Seghier ML, Price CJ: Where, when and why brain activation differs for bilinguals and monolinguals during picture naming and reading aloud. Cereb Cortex 2011, doi: 10.1093/cercor/bhr161.

57. Miura N, Iwata K, Watanabe J, Sugiura M, Akitsuki Y, Sassa Y, Ikuta N, Okamoto H, Watanabe Y, Riera J, Maeda Y, Matsue Y, Kawashima R: Cortical activation during reading aloud of long sentences: fMRI study. Neuroreport 2003, 14:1563-1566.

58. Miura N, Watanabe J, Iwata K, Sassa Y, Riera J, Tsuchiya H, Sato S, Horie K, Takahashi M, Kitamura M, Kawashima R: Cortical activation during reading of ancient versus modern Japanese texts: fMRI study. Neuroimage 2005, 26:426-431.

59. Arsalidou M, Taylor MJ: Is $2+2=4$ ? Meta-analyses of brain areas needed for numbers and calculations. Neuroimage 2011, 54:2382-2393.

60. Kawashima R, Taira M, Okita K, Inoue K, Tajima N, Yoshida H, Sasaki T, Sugiura M, Watanabe J, Fukuda H: A functional MRI study of simple arithmetic-a comparison between children and adults. Brain Res Cogn Brain Res 2004, 18:227-233.

61. Menon V, Rivera SM, White CD, Glover GH, Reiss AL: Dissociating prefrontal and parietal cortex activation during arithmetic processing. Neuroimage 2000, 12:357-365.

62. Dubois B, Slachevsky A, Litvan I, Pillon B: The FAB: a Frontal Assessment Battery at bedside. Neurology 2000, 55:1621-1626.

63. Kugo A, Terada S, Ata T, Ido Y, Kado Y, Ishihara T, Hikiji M, Fujisawa Y, Sasaki K, Kuroda S: Japanese version of the Frontal Assessment Battery for dementia. Psychiatry Res 2007, 153:69-75.

64. Nakaaki S, Murata Y, Sato J, Shinagawa Y, Matsui T, Tatsumi H, Furukawa TA Reliability and validity of the Japanese version of the Frontal Assessment Battery in patients with the frontal variant of frontotemporal dementia. Psychiatry Clin Neurosci 2007, 61:78-83.

65. Wechsler DA: Wechsler Adult Intelligence Scale. 3 edition. San Antonio, TX: The Psychological Corporation; 1997.

66. Schulz KF, Altman DG, Moher D: CONSORT 2010 Statement: updated guidelines for reporting parallel group randomised trials. Trials 2010, 11:32.

67. Matsuoka K, Uno M, Kasai K, Koyama K, Kim Y: Estimation of premorbid IQ in individuals with Alzheimer's disease using Japanese ideographic script (Kanji) compound words: Japanese version of National Adult Reading Test. Psychiatry Clin Neurosci 2006, 60:332-339.

68. Clark F, Azen SP, Zemke R, Jackson J, Carlson M, Mandel D, Hay J, Josephson K, Cherry B, Hessel C, Palmer J, Lipson L: Occupational therapy for independent-living older adults. A randomized controlled trial. JAMA 1997, 278:1321-1326

69. Hakoda Y, Sasaki M: Group version of the Stroop and reverse-Stroop test: The effects of reaction mode, order and practice. Kyoiku Shinrigaku Kenkyu (JPN J Educ Psychol) 1990, 38:389-394.

70. Ito $E$, Hatta T, Ito $Y$, Kogure T, Watanabe H: Performance of verbal fluency tasks in Japanese healthy adults: Effect of gender, age and education on the performance. Shinkei Shinrigaku Kenkyu (JPN J Neuropsychol) 2004, 20:254-263.

71. Wechsler DA: Wechsler Memory Scale Revised San Antonio, TX: The Psychological Corporation; 1987.

72. Wilson BA, Cockburn J, Baddeley AD: The Rivermead Behavioral Memory Test Reading: Thames Valley Test Company; 1985.

73. Hatta T, Ito Y, Yoshizaki K: D-CAT manual (Screening test for attention) Osaka: Union Press; 2000

74. Alvarez JA, Emory E: Executive function and the frontal lobes: a metaanalytic review. Neuropsychol Rev 2006, 16:17-42.

75. Goldberg E, Bougakov D: Neuropsychologic assessment of frontal lobe dysfunction. Psychiatr Clin North Am 2005, 28:567-580, 578-579.

76. Watanabe M, Hakoda Y, Matsumoto A: Group Version of the Stroop and Reverse-Stroop Test: An Asymmetric developmental trait in two kinds of 
interference. Kyusyu Daigaku Shinrigaku Kenkyu (Kyushu University

Psychological Research) 2011, 12:41-50.

77. Nelson HE: National Adult Reading Test (NART) Windsor: NFER-Nelson; 1982

78. Faul F, Erdfelder E, Buchner A, Lang AG: Statistical power analyses using

G*Power 3.1: tests for correlation and regression analyses. Behav Res Methods 2009, 41:1149-1160.

79. Faul F, Erdfelder E, Lang AG, Buchner A: G*Power 3: a flexible statistical power analysis program for the social, behavioral, and biomedical sciences. Behav Res Methods 2007, 39:175-191.

80. Cohen J: Statistical power analysis for the behavioral sciences. 2 edition. Hillsdale, NJ: L. Erlbaum Associates; 1988.

81. Dempster AP, Laird NM, Rubin DB: Maximum likelihood from incomplete data via the EM algorithm. J Roy Stat Soc B Stat Meth 1977, 39:1-38.

82. Basak C, Boot WR, Voss MW, Kramer AF: Can training in a real-time strategy video game attenuate cognitive decline in older adults? Psychol Aging 2008, 23:765-777.

83. Berry AS, Zanto TP, Clapp WC, Hardy JL, Delahunt PB, Mahncke HW, Gazzaley A: The influence of perceptual training on working memory in older adults. PLoS One 2010, 5:e11537.

84. Li KZ, Roudaia E, Lussier M, Bherer L, Leroux A, McKinley PA: Benefits of cognitive dual-task training on balance performance in healthy older adults. J Gerontol A Biol Sci Med Sci 2010, 65:1344-1352.

85. Czaja SJ, Sharit J: Age differences in the performance of computer-based work. Psychol Aging 1993, 8:59-67.

86. Czaja SJ, Sharit J: Age differences in attitudes toward computers. $J$ Gerontol B Psychol Sci Soc Sci 1998, 53:P329-P340.

87. Sharit J, Czaja SJ: Ageing, computer-based task performance, and stress: issues and challenges. Ergonomics 1994, 37:559-577.

doi:10.1186/1745-6215-13-32

Cite this article as: Nouchi et al:: Beneficial effects of reading aloud and solving simple arithmetic calculations (learning therapy) on a wide range of cognitive functions in the healthy elderly: study protocol for a randomized controlled trial. Trials 2012 13:32.

\section{Submit your next manuscript to BioMed Central and take full advantage of:}

- Convenient online submission

- Thorough peer review

- No space constraints or color figure charges

- Immediate publication on acceptance

- Inclusion in PubMed, CAS, Scopus and Google Scholar

- Research which is freely available for redistribution

Submit your manuscript at www.biomedcentral.com/submit 